the impression that endogenous $\mathrm{CO}$ production predisposes $\mathrm{pregnant}$ women to $\mathrm{CO}$ intoxication.

Another weakness of the book is probably due to the policy of the publisher: to widen the possible circle of readers as far as possible by giving some background material. Information like "In man, the kidneys are bean-shaped structures about 4 in long" and that "Renal arteries and veins are supplied to the kidneys" somewhat underestimate the intelligence of the non-science graduate readers, who are

\section{Development in mammals}

Developments in Mammals. Vol. 3. Edited by M. H. Johnson. Pp.472. (North-Holland: Amsterdam, New York and Oxford, 1978.) \$61; Df. 140.

IN many respects this third volume of Development of Mammals is the best so far, offering several excellent reviews of topics which have only recently undergone a burst of stimulating activity. On the other hand, some of the reviews are not without doses of repetition and excessive speculation, and would have benefited from a firmer editorial hand. In the preface, Martin Johnson tells us that he encouraged his authors "to develop new ideas and to be provocative", but sometimes the provocation is of the wrong kind! All in all, however, this is a good volume, and one to which developmental biologists of both the molecular biology and pattern formation schools may turn with interest.

Appropriately, the volume begins with two articles by Meizel and by Green on the act that starts it allfertilisation-or, rather, on the preview to Act One, in which the sperm undergoes the acrosome reaction. The acrosome is a giant secretory granule lying near the sperm tip, and the membrane of the acrosome fuses with the overlying plasma membrane so that the store of hydrolytic enzymes is exposed. The exact stimulus for the reaction, the mechanism of membrane fusion, and the function of the enzymes are all unknown, and the two articles debate the available evidence.

Recent work on monoclonal antibodies reacting with murine teratocarcinoma cells and early mouse embryos has made the review by Pratt on membrane lipids of embryos a timely contribution. At least one, and probably several, of these monoclonal antibodies recognises a glycolipid, and not a few developmental biologists have been embarrassed to discover their ignorance about this class of molecule. supposed to understand quite complex biochemical processes which are explained at a significantly higher level.

However, compared with the valuable information and pioneer spirit, this criticism is only of minor significance and it would be wrong if this diverted people interested in environmental heaith from reading the book and thinking about the problems

\section{Laszlo Magos}

Laszlo Magos is at the MRC Toxicology Unit, Carshalton, UK.

The role of virus-like particles in early embryos is discussed by Daniel and Chilton, and this is followed by an excellent review by Monk of Xchromosome inactivation in female embryos. This topic is one which has undergone a recent flurry of experimentation, and the author combines the new data with speculation in a nice, economical style.

Teratocarcinoma cells receive their fair share of mention in this volume, and Martin tackles the difficulties as well as the advantages of using in vitro cultures of these cells as a model for mammalian development. Her appraisal of the results from several laboratories on the fate of teratocarcinoma cells injected into host blastocysts should be required reading for all molecular biologists who now believe that a normal mouse can be generated from teratocarcinoma cells with, as Martin puts it, "a flick of the micromanipulator".

The origin of the primordial germ cells, their migration from the yolk sac

\section{Cell proliferation and the cell cycle}

Cell Cycle Regulation. Edited by J. R. Jeter, I. L. Cameron, G. M. Padilla and A. M. Zimmerman. Pp. 255. (Academic: New York and London, 1978.) \$23; £14.95.

MANy texts on the cell cycle confuse (wisely?) progression through the cell cycle of continuously replicating cells with the control of cell proliferation in growing populations. This book is largely concerned with the regulation of the progression through the cell cycle in cycling cells, and consists of eleven separate contributions. McCarty reviews recent progress in chromatin structure and surveys in a general way the post-synthetic modifications of chromatin proteins. In no case is the precise molecular function of any of these modifications known; although there is recent and exciting progress in the study of acetylation, phos- along the gut and into the genital ridge, and the influence of the somatic cells of the gonad on germ cell maturation and secondary sexual organ development are discussed in articles by Heath, O, and Donahoe and Swann.

The remaining articles move on to the wider arena of morphogenesis in the mammalian embryo. Looking at the incredible scanning electron micrographs of neurula stage embryos presented in this volume one can only admire scientists like Snow, and Morriss and Thorogood, who have begun the painstaking task of analysing the forces responsible for generating such complex, three-dimensional shapes. One of the most stimulating ideas in this field, put forward recently by Snow and discussed in his review, is that all the tissues of the mammalian foetus are derived from a small group of rapidly dividing cells, or proliferation centre, in the early epiblast. Testing this hypothesis, and following clonal growth and cell migration, would be much easier if there were a genetic marker in the mouse detectable intracellularly early in development. As it is, the final article by West reviews the information obtained so far using chimaeras between mouse embryos differing in genetic markers expressed late in development.

The book is well produced, although the type is too small for comfort, and the articles are well referenced.

Brigid Hogan

Brigid Hogan is a member of the scientific staff at the Imperial Cancer Research Fund Laboratories, Mill Hill, London, $U K$.

phorylation and ADP-ribosylation.

Gurley et al. give a detailed description of their work on phosphorylation through the cell cycle of CHO cells. The phosphorylation of histone $\mathrm{H} 1$ in $G_{1}$, in $S$ and a "superphosphorylation" at the time of mitosis seems to be generally true. They discuss the controversy with Matthews and Bradbury as to whether superphosphorylation is associated with condensed chromosomes, or is a preceding "trigger event".

Krause discusses the variable binding of histones during the cell cycle. Harris brings together information on microtubules, membranes vesicles and calcium to construct an hypothesis for the initiation of mitosis. Gerson reviews the possible role of intracellular $p \mathrm{H}$ in the cell cycle. Recent interest in the possible regulatory functions of such parameters as $p \mathrm{H}$ and divalent cation availability revives hypotheses of some 30 years ago.

Physarum rears its beautiful heads twice in this book; it is (of course) the ideal eukaryotic organism for cell-cycle 